\title{
ADMITTANCE MATRIX MODEL OF A SYNCHRONOUS MACHINE FOR HARMONIC ANALYSIS
}

\author{
A. Semlyen \\ University of Toronto \\ Toronto, Ontario, Canada
}

\author{
J.F. Eggleston J. Arrillaga \\ University of Canterbury \\ Christchurch, New Zealand
}

\begin{abstract}
The harmonic analysis of a power system requires appropriate models of all system components. Synchronous machines act as harmonic converters, sensitive to the sequence of the fundamental and harmonic frequencies. This paper describes the derivation of a harmonic model of the machine in the form of a three-phase complex admittance matrix and its application to the harmonic behaviour of an asymmetrically loaded generator.
\end{abstract}

\section{INTRODUCTION}

The importance of harmonic analysis of power systems is on the rise [1] due to an increased use of converters which are the primary source of harmonics. Several papers have been published on this topic, some describing the problem as harmonic power flow $[2],[3]$, some as harmonic penetration [4], and a recent book deals with power system harmonics [5]. In addition to converters, also arc furnaces, fluorescent lamps and the magnetizing branches of transformers [6] are recognized sources of harmonics. However, the harmonic behaviour of the synchronous generator has not been given serious consideration in harmonic analysis [4], even though it is well known that it converts negative sequence currents into third harmonic positive sequence and, in general, acts as a harmonic converter. This is so probably because of the complexity of the problem, since conversion means coupling of harmonics which otherwise would be examined separately. Therefore, generators are often represented by a single approximate impedance at each harmonic. However, three-phase transmission lines appear as strongly unbalanced at harmonic frequencies and resonances may appear for single modes so that harmonic unbalance is created or strongly amplified [7]. The generator will pick up the unbalance and return other harmonics. Clearly, the complex interaction between generator and system cannot be ignored.

This paper describes the derivation of a synchronous machine model and its application to the analysis of an asymmetrically loaded synchronous generator. It is a three-phase model because of the significance of negative sequence in harmonic conversion. It contains all harmonics (even and odd, as these turn out to be uncoupled) and permits the calculation of the current vector

$$
\mathbf{i}=\mathbf{Y}_{a b c} \mathbf{v}
$$

Data for synchronous machines are normally available in terms of $d-q$ axis and implicit in this information is the effect of the second harmonic terms of the inductances. Information on fourth harmonic terms, absent from the $d-q$ axis model, is not generally available and is ignored in this investigation.

It is also assumed that a base load flow solution has been obtained for the power system, balanced out at the fundamental frequency, so that a standard load flow program can be used. The harmonic analysis is performed subsequently on the unbalanced system in a three-phase representation. The results of the harmonic

36 SM 35()-3 A paper recommended and approved by the LEEE Power System Fngineering Committee of the IEFE Power Engineering Society for presentation at the IFEE/PFS 1986 Sumer Meeting, Mexico City, Mexico, July $20-25 ; 1986$. Manuscript submitted January 18, 1985; made available for printing May $5,1986$.

Printed in the U.S.A. analysis are superimposed on the base load flow solution and represent an increment to it. The harmonic analysis will contain all significant frequencies, including d.c. and the fundamental frequency. The reference of the latter serves also as a phase reference for the harmonics. Since the harmonic analysis is superimposed on the base load flow, the generator will be assumed as having a shortcircuited field winding, which makes the harmonic model completely passive. Equation (1) corresponds to this condition.

\section{HARMONIC MODEL OF A SYNCHRONOUS MACHINE}

\section{Derivation of the Matrix $\mathbf{Y}_{\mathrm{dq}}$}

The synchronous machine admittance model is derived from the $d, q$-axis differential equations. Generally accepted conventions and notations are used, as detailed for instance in Ref. [8] and illustrated in Fig. 1. With two damper windings in the rotor, $s$ and $t$, the equations are:

$$
\begin{gathered}
v_{d}=R i_{d}+p\left(L_{d} i_{d}+M_{d f} i_{f}+M_{d s} i_{s}\right)-\omega\left(L_{q} i_{q}+M_{q t} i_{t}\right) \\
\left.v_{q}=R i_{q}+p\left(L_{q} i_{q}+M_{q t} i_{t}\right)+\omega\left(L_{d} i_{d}+M_{d f} i_{f}+M_{d s} i_{s}\right)^{\prime}\right) \\
v_{f}=R_{f} i_{f}+p\left(L_{f} i_{f}+M_{d f} i_{d}+M_{f s} i_{s}\right)=0 \\
v_{s}=R, i_{s}+p\left(L_{s} i_{s}+M_{d s} i_{d}+M_{f s} i_{f}\right)=0 \\
v_{t}=R_{t} i_{t}+p\left(L_{t} i_{t}+M_{q t} i_{q}\right)=0
\end{gathered}
$$

In (2) $v_{f}$ has been set to zero because, as mentioned, the field voltage has been considered in the base load flow. However, the parameters of the excitation circuit have to be included in $R_{f}$ and $L_{f}$. All variables are phasors of harmonic $h$ :

$$
w_{h}=\operatorname{Re}\left\{\mathbf{W}_{h} e^{j h \omega t}\right\}=W^{\prime}{ }_{h} \cos (h \omega t)-W^{\prime \prime}{ }_{h} \sin (h \omega t)
$$

where

$$
\mathbf{W}_{h}=W_{h}^{\prime}+j W^{\prime \prime}
$$

denotes any phasor. Bold is used to denote a complex number and/or a matrix or vector. We have now

$$
\mathbf{p}=j h \omega
$$

and equations (2) become algebraic operations in $V_{d_{h}}, V_{q_{h}}, I_{d_{h}}, I_{q_{h}}$, $\mathbf{I}_{f_{h}}, \mathbf{I}_{s_{h}}, \mathbf{I}_{t_{h}}$. The last three can be eliminated from (2'), using (2") so that we obtain

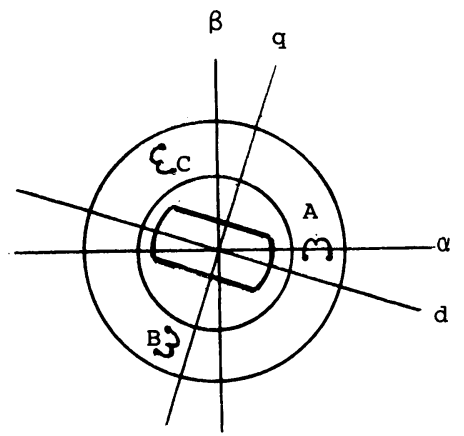

Figure 1 Basic machine representation 


$$
\left[\begin{array}{c}
\mathbf{V}_{d_{h}} \\
\mathbf{V}_{q_{h}}
\end{array}\right]=\left[\begin{array}{ll}
\mathbf{Z}_{1_{h}} & \mathbf{Z}_{\mathbf{2}_{h}} \\
\mathbf{Z}_{3_{h}} & \mathbf{Z}_{\mathbf{4}_{h}}
\end{array}\right]\left[\begin{array}{l}
\mathbf{I}_{d_{h}} \\
\mathbf{I}_{q_{h}}
\end{array}\right]
$$

or, in more compact form

$$
\mathbf{v}_{d q_{h}}=\mathbf{Z}_{d q_{h}} \mathbf{i}_{d q_{h}}
$$

and

$$
\mathbf{i}_{d q_{h}}=\mathbf{Y}_{d q_{h}} \mathbf{v}_{d q_{h}}
$$

For simplicity, we will denote the elements of the nonsymmetric matrices $\mathbf{Z}_{d q_{h}}$ or $\mathbf{Y}_{d q_{h}}$ by

$$
\left[\begin{array}{ll}
\mathbf{a}_{h} & \mathbf{b}_{h} \\
\mathbf{c}_{h} & \mathbf{d}_{h}
\end{array}\right]
$$

A particular matrix $\mathbf{Z}_{d q_{h}}$ of interest is for the rotor frequency $h=0$. Then $p$ becomes zero and equations (2') yield

$$
\mathbf{z}_{d q_{o}}=\left[\begin{array}{cc}
R & -\omega L_{q} \\
\omega L_{d} & R
\end{array}\right]
$$

\section{Derivation of Connection Matrix C}

Although our aim is to derive an admittance matrix in phase quantities, the mathematical formulation is simplified by using an intermediate connection matrix in terms of $\alpha, \beta$ quantities, introduced by the rotation

$$
\left[\begin{array}{l}
v_{\alpha_{h^{\prime}}} \\
v_{\beta_{h^{\prime}}}
\end{array}\right]=\left[\begin{array}{cc}
\cos \omega t & -\sin \omega t \\
\sin \omega t & \cos \omega t
\end{array}\right]\left[\begin{array}{l}
v_{d_{h}} \\
v_{q_{h}}
\end{array}\right]
$$

where $h^{\prime}$ denotes the resultant harmonics. The inverse transformation is

$$
\left[\begin{array}{c}
v_{d_{h^{\prime}}} \\
v_{q_{h^{\prime}}}
\end{array}\right]=\left[\begin{array}{cc}
\cos \omega t & \sin \omega t \\
-\sin \omega t & \cos \omega t
\end{array}\right]\left[\begin{array}{c}
v_{\alpha_{h}} \\
v_{\beta_{h}}
\end{array}\right]
$$
(3),

Identical transformation applies for currents. If we write, as in

$$
\begin{aligned}
& v_{d_{h}}=V_{d_{h}}^{\prime} \cos (h \omega t)-V^{\prime \prime}{ }_{d_{h}} \sin (h \omega t) \\
& v_{q_{h}}=V_{q_{h}}^{\prime} \cos (h \omega t)-V^{\prime \prime}{ }_{q_{h}} \sin (h \omega t)
\end{aligned}
$$

and substitute (10) into (9'), we obtain expressions for $v_{\alpha_{h^{\prime}}}$ and $v_{\beta_{h^{\prime}}}$ in the form of (3), with $h^{\prime}=h \pm 1$ :

$$
\begin{aligned}
v_{\alpha_{h^{\prime}}}= & V_{\alpha_{h-1}^{\prime}}^{h} \cos (h-1) \omega t-V^{\prime \prime}{ }_{\alpha_{h-1}}^{h} \sin (h-1) \omega t+ \\
& +V_{\alpha_{h+1}^{\prime}}^{h} \cos (h+1) \omega t-V_{\alpha_{h+1}^{\prime \prime}}^{h} \sin (h+1) \omega t \\
v_{\beta_{h^{\prime}}}= & V_{\beta_{h-1}^{\prime}}^{h} \cos (h-1) \omega t-V_{\beta_{h-1}^{\prime \prime}}^{h} \sin (h-1) \omega t+ \\
& +V_{\beta_{h+1}^{\prime}}^{h} \cos (h+1) \omega t-V_{\beta_{h+1}^{\prime \prime}}^{h} \sin (h+1) \omega t
\end{aligned}
$$

where

$$
\begin{aligned}
V_{\alpha_{h-1}}^{\prime h}=\frac{1}{2}\left(V_{d_{h}}^{\prime}+V_{q_{h}}^{\prime \prime}\right) & V_{\alpha_{h-1}^{\prime \prime}}^{h}=\frac{1}{2}\left(V_{d_{h}}^{\prime \prime}-V_{q_{h}}^{\prime}\right) \\
V_{\alpha_{h+1}}^{\prime h}=\frac{1}{2}\left(V_{d_{h}}^{\prime}-V_{q_{h}}^{\prime \prime}\right) & V^{\prime \prime}{ }_{\alpha_{h+1}}^{h}=\frac{1}{2}\left(V_{d_{h}}^{\prime \prime}+V_{q_{h}}^{\prime}\right)
\end{aligned}
$$

$$
\begin{aligned}
& \text { and } \\
& \begin{array}{ll}
V_{\beta_{h-1}}^{h}=\frac{1}{2}\left(-V_{d_{h}}^{\prime \prime}+V_{q_{h}}^{\prime}\right) & V_{\beta_{h-1}}^{\prime \prime h}=\frac{1}{2}\left(V_{d_{h}}^{\prime}+V_{q_{h}}^{\prime \prime}\right) \\
V_{\beta_{h+1}}^{\prime h}=\frac{1}{2}\left(V_{d_{h}}^{\prime \prime}+V_{q_{h}}^{\prime}\right) & V_{\beta_{h+1}^{\prime \prime}}^{\prime \prime}=\frac{1}{2}\left(-V_{d_{h}}^{\prime}+V_{q_{h}}^{\prime \prime}\right)
\end{array}
\end{aligned}
$$

These can be combined into phasors, as in (4), yielding:

$$
\begin{aligned}
& \mathrm{V}_{\alpha_{h-1}}^{h}=\frac{1}{2}\left(\mathrm{~V}_{d_{h}}-j \mathrm{~V}_{q_{h}}\right) \\
& \mathrm{V}_{\alpha_{h+1}}^{h}=\frac{1}{2}\left(\mathrm{~V}_{d_{h}}+j \mathrm{~V}_{q_{h}}\right)
\end{aligned}
$$

and

$$
\begin{aligned}
& \mathrm{V}_{\beta_{h-1}}^{h}=\frac{1}{2}\left(j \mathrm{~V}_{d_{h}}+\mathrm{V}_{q_{h}}\right) \\
& \mathbf{V}_{\beta_{h+1}}^{h}=\frac{1}{2}\left(-j \mathrm{~V}_{d_{h}}+\mathrm{V}_{q_{h}}\right)
\end{aligned}
$$

In equations (11), (12) and (13) the superscript $h$ indicates the related $d, q$-axis harmonic for a component of order $h \pm 1$ of a stator $(\alpha, \beta$-axis) harmonic. When two such components of the same order (related to a lower and a higher order rotor harmonic) combine, the superscript is dropped; see the vector $\left(16^{\prime}\right)$.

Equations (13) should be written for all $h$. However if $h=0$ or $h-1=0$, then $\mathrm{V}_{o}=V_{o} ;$ and if $h=0$, then we obtain directly from $\left(9^{\prime}\right)$, converted into phasor relations,

$$
\begin{aligned}
& \mathbf{V}_{\alpha_{1}}=V_{d_{o}}+j V_{q_{0}} \\
& \mathbf{V}_{\beta_{1}}=-j V_{d_{0}}+V_{q_{0}}
\end{aligned}
$$
obtain

By assembling equations (13) and taking (14) into account, we

$$
\mathbf{v}_{\alpha \beta}=\mathbf{C} \mathbf{v}_{d q}
$$

An identical transformation applies for currents:

$$
\mathbf{i}_{\alpha \beta}=\mathbf{C i}_{d q}
$$

In (15')

$$
\begin{aligned}
& \mathbf{v}_{\alpha \beta}=\left[V_{\alpha_{o}}, V_{\beta_{0}}, \mathbf{v}_{\alpha_{1}}, \mathbf{v}_{\beta_{1}}, \mathbf{v}_{\alpha_{2}}, \mathbf{v}_{\beta_{2}}, \ldots, \mathbf{v}_{\alpha_{n}}, \mathbf{v}_{\beta_{n}}\right]^{T} \\
& \mathbf{v}_{d q}=\left[V_{d_{0}}, V_{q_{0}}, \mathbf{v}_{d_{1}}, \mathbf{v}_{q_{1}}, \mathbf{v}_{d_{2}}, \mathbf{v}_{q_{2}}, \ldots, \mathbf{v}_{d_{n}}, \mathbf{v}_{q_{n}}\right]^{T}
\end{aligned}
$$

and

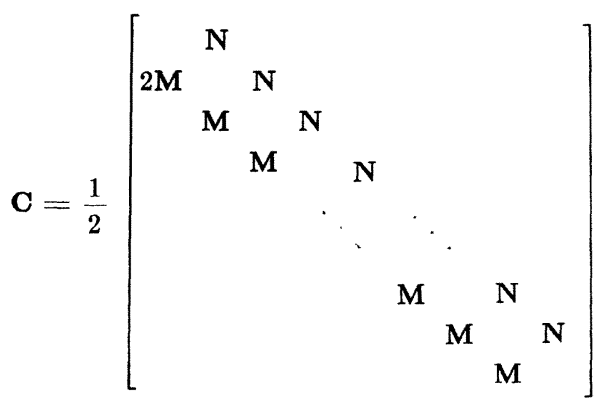

with 
where the asterisk denotes the conjugate and $n$ the highest harmonic investigated.

The matrix $\mathbf{C}$ is singular because it contains pairs of rows (or columns) with matrices $\mathbf{M}$ or $\mathbf{N}$ only, which are singular due to the fixed relation between the $\alpha$ and $\beta$, or the $d$ and $q$ components, respectively. This singularity can be removed by deleting the first (or the second) row and column of the matrix and by leting its size increase indefinitely (or, alternatively, deleting also the last row and column). In this way, only one of $V_{\alpha_{o}}, V_{\beta_{a}}$ and of $V_{d_{o}}, V_{q_{0}}$ will remain in each of the vectors $\mathbf{v}_{\alpha \beta}$ and $\mathbf{v}_{d q}$ of (16) (and only one of the last two elements in these vectors if the truncated matrix $\mathbf{C}$ is considered). Keeping only one of $V_{a_{0}}, V_{\beta_{0}}$ does not result in loss of information since $V_{\beta_{0}}=j V_{\alpha_{0}}$. Similarly, it can be seen from (17') that the sum $V_{d_{0}}+j V_{q_{o}}$ itself affects the value of $\mathbf{V}_{\alpha_{1}}$ and of $\mathrm{V}_{\beta_{1}}$, rather than its individual components $V_{d_{0}}$ and $V_{q_{0}}$. Any of the latter, having assigned an appropriate complex value, can replace the sum $V_{d_{0}}+j V_{q_{0}}$.

Let $\mathbf{C}^{\prime}$ denote the reduced matrix $\mathbf{C}$, and let $\mathbf{C}^{* *}$ and $\mathbf{C}^{*}$ be their conjugate, respectively. Then it can be easily verified, by direct substitution and taking (17") into account, that $\mathbf{C}^{\prime} \mathbf{C}^{*}=\mathbf{U}$, where $U$ is the unity matrix. This does not mean however that $\mathbf{C}^{*}$ is the inverse of $\mathbf{C}$. Still, we prefer to use the full matrix $\mathbf{C}$ of (17') because of its symmetry and simplicity, with the understanding that a reference to an inverse relationship to (15) is interpreted to apply to the reduced matrix $\mathbf{C}^{\prime}$ and the reduced set of variables. The inverse relationship to (15) can be derived directly starting with equations (9") and performing calculations as in equations (10) to (14). We obtain

$$
\begin{aligned}
& \mathbf{v}_{d q}=\mathbf{C}^{*} \mathbf{v}_{\alpha \beta} \\
& \mathbf{i}_{d q}=\mathbf{C}^{*} \mathbf{i}_{\alpha \beta}
\end{aligned}
$$

\section{Derivation of Matrix $\mathbf{Y}_{\alpha \beta}$}

We assemble equations (7"), for all $h$, into the relation:

$$
\mathbf{i}_{d q}=\mathbf{Y}_{d q} \mathbf{v}_{d q}
$$

where $\mathbf{Y}_{d q}$ is block diagonal with blocks of the form (8).

For given $v_{\alpha \beta}$ we obtain $v_{d q}$ from (18') then $i_{d q}$ from (19) and $\mathbf{i}_{\alpha \beta}$ from (15"). Alternatively, we can write

$$
\mathbf{i}_{\alpha \beta}=\mathbf{Y}_{\alpha \beta} \mathbf{v}_{\alpha \beta}
$$

where

$$
\mathbf{Y}_{\alpha \beta}=\mathbf{C} \mathbf{Y}_{d q} \mathbf{C}^{*}
$$

The non-symmetric matrix $\mathbf{Y}_{\alpha \beta}$ has the form

$$
\mathbf{Y}_{\alpha \beta}=\left[\begin{array}{llllllll}
\mathbf{A}_{0} & & \mathbf{B}^{\prime}{ }_{1} & & & & & \\
& \mathbf{A}_{1} & & \mathbf{B}^{\prime}{ }_{2} & & & \\
\mathbf{B}^{\prime}{ }_{1} & & \mathbf{A}_{2} & & \ddots & & & \\
& \mathbf{B}^{\prime}{ }_{2} & & \ddots & \mathbf{B}^{\prime \prime} & & \\
& & \ddots & & \mathbf{A}_{h} & & \ddots & \\
& & \mathbf{B}^{\prime}{ }_{n} & & \ddots & & \mathbf{B}^{\prime}{ }_{n-1} \\
& & & \ddots & & \mathbf{A}_{n-1} & \\
& & & & \mathbf{B}^{\prime}{ }_{n-1} & & \mathbf{A}_{n}
\end{array}\right]
$$

where

$$
\begin{aligned}
& \mathbf{A}_{0}=\mathbf{N} \mathbf{Y}_{d q_{1}} \mathbf{N} / 2 \\
& \mathbf{A}_{1}=\left(2 \mathbf{M Y}_{d q_{0}} \mathbf{M}+\mathbf{N} \mathbf{Y}_{d q_{2}} \mathbf{N}\right) / 4 \\
& \mathbf{A}_{h}=\left(\mathbf{M Y _ { d q _ { h - 1 } }} \mathbf{M}+\mathbf{N Y _ { d q _ { h + 1 } }} \mathbf{N}\right) / 4(\text { for } h=2, \ldots, n-1)
\end{aligned}
$$

$$
\begin{aligned}
& \mathbf{B}^{\prime}{ }_{1}=\mathbf{M Y} \mathbf{Y}_{d q_{1}} \mathbf{N} / 2 \\
& \mathbf{B}^{\prime}{ }_{h}=\mathbf{M} \mathbf{Y}_{d q_{h}} \mathbf{N} / 4 \\
& \mathbf{B}^{\prime \prime}{ }_{h}=\mathbf{N} \mathbf{Y}_{d q_{h}} \mathbf{M} / 4
\end{aligned}
$$$$
\text { (for } h=2, \ldots, n-1)
$$$$
\text { (for } h=1, \ldots, n-1)
$$

$$
\mathbf{A}_{n}=\mathbf{M} \mathbf{Y}_{d q_{n-1}} \mathbf{M} / 4
$$

These expressions can be calculated from:

$$
\begin{aligned}
& \mathbf{M} \mathbf{Y}_{d q_{i}} \mathbf{M}=\left(\left(\mathbf{a}_{i}+\mathbf{d}_{i}\right)-j\left(\mathbf{b}_{i}-\mathbf{c}_{i}\right)\right) \mathbf{M} \\
& \mathbf{N} \mathbf{Y}_{d q_{i}} \mathbf{N}=\left(\left(\mathbf{a}_{i}+\mathbf{d}_{i}\right)+j\left(\mathbf{b}_{i}-\mathbf{c}_{i}\right)\right) \mathbf{N} \\
& \mathbf{M} \mathbf{Y}_{d q_{i}} \mathbf{N}=\left(\left(\mathbf{a}_{i}-\mathbf{d}_{i}\right)+j\left(\mathbf{b}_{i}+\mathbf{c}_{i}\right)\right) \mathbf{P} \\
& \mathbf{N} \mathbf{Y}_{d q_{i}} \mathbf{M}=\left(\left(\mathbf{a}_{i}-\mathbf{d}_{i}\right)-j\left(\mathbf{b}_{i}+\mathbf{c}_{i}\right)\right) \mathbf{Q}
\end{aligned}
$$

where

$$
\mathbf{P}=\left[\begin{array}{cc}
1 & -j \\
-j & -1
\end{array}\right], \quad \mathbf{Q}=\left[\begin{array}{cc}
1 & j \\
j & -1
\end{array}\right]
$$

From equations (23) we can see that the self admittance $\mathbf{A}_{h}$ for the harmonic of order $h$ depends only on the rotor frequencies of order $h \pm 1$, and the mutuals $\mathbf{B}{ }^{\prime}{ }_{h}$ and $\mathbf{B}{ }^{\prime}{ }_{h}$ between harmonics $h+1$ and $h-1$ depend on the rotor frequency of order $h$. These results are consistent with the well known pertinent physical phenomena. An intuitive block diagram of the voltage and current harmonic relationships in the armature and rotor is shown in Figure

The matrix $\mathbf{Y}_{\alpha \beta}$ has one non-zero block band on each side of the diagonal and a zero block separating band. Therefore even and odd harmonics do not interact in the generator.

The impedance matrix $Z_{\alpha \beta}$ can be obtained similarly to (21):

$$
\mathbf{Z}_{\alpha \beta}=\mathbf{C} \mathbf{Z}_{d q} \mathbf{C}^{*}
$$

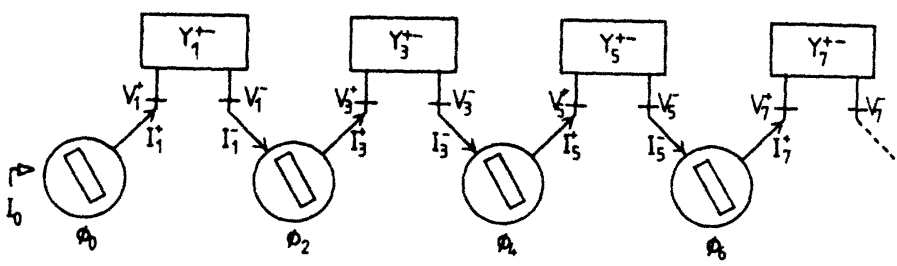

Figure 2 Harmonic interaction between generator and system Derivation of Matrix $Y_{a b c}$

We first augment $Y_{\alpha \beta}$ by a zero sequence diagonal matrix, comprising all harmonics:

$$
\mathbf{Y}_{\alpha \beta o}=\left[\begin{array}{ll}
\mathbf{Y}_{\alpha \beta} & \\
& \mathbf{Y}_{o}
\end{array}\right]
$$

It is generally assumed that zero sequence currents produce no significant resultant flux in the air-gap and accordingly equation (27) shows no coupling between the zero sequence and the $\alpha, \beta$ components. At least one author [9] discuinis briefly such an effect, but stresses that it is negligible and subsequently removes the coupling from the analysis. 
For any harmonic we can write

$$
\begin{aligned}
& \mathbf{v}_{\alpha \beta o_{h}}=\mathbf{T}_{h} \mathbf{v}_{a b c_{h}} \\
& \mathbf{i}_{\alpha \beta o_{h}}=\mathbf{T}_{h} \mathbf{i}_{a b c_{h}}
\end{aligned}
$$

where

$$
\mathbf{T}_{h}=\left[\begin{array}{ccc}
1 & -\frac{1}{2} & -\frac{1}{2} \\
0 & \frac{\sqrt{3}}{2} & -\frac{\sqrt{3}}{2} \\
\frac{1}{3} & \frac{1}{3} & \frac{1}{3}
\end{array}\right]
$$

Equations (27), (28) and (29) can be completed and assembled to yield the following:

$$
\begin{aligned}
& \mathbf{v}_{\alpha \beta o}=\mathbf{T} \mathbf{v}_{a b c} \\
& \mathbf{i}_{\alpha \beta o}=\mathbf{Y}_{\alpha \beta o} \mathbf{v}_{\alpha \beta o} \\
& \mathbf{i}_{a b c}=\mathbf{T}^{-1} \mathbf{i}_{\alpha \beta o}
\end{aligned}
$$

An admittance matrix

$$
\mathbf{Y}_{a b c}=\mathbf{T}^{-1} \mathbf{Y}_{\alpha \beta \beta} \mathbf{T}
$$

could be calculated, but the sequence $(30)$ is equivalent to the intended use in (1) (where the subscripts $a b c$ have been omitted from $v$ and $i$ ).

\section{APPLICATION OF THE MODEL TO AN ASYMMETRICALLY LOADED GENERATOR}

The test system of Figure 3 is used to illustrate the effect of the harmonic admittance model of the generator. It consists of a single circuit line of flat configuration corresponding to the Islington to Kikiwa $220 \mathrm{kV}$ transmission line in the New Zealand system [7]. The transformer is $Y_{g} / Y_{g}$ connected, and its rating is $100 \mathrm{MVA}$, $14 \mathrm{kV} / 220 \mathrm{kV}$, with a leakage reactance of 0.112 p.u.

Equation (1) is modified to include the effect of the fundamental power flow solution. This is achieved by adding a positive sequence fundamental current injection $i_{o}$ which maintains a constant (1 p.u.) positive sequence component of fundamental voltage at the generator terminals. Taking into account the sign convention indicated in Figure 3, the generator current becomes

$$
\mathbf{i}=-\mathbf{Y}_{\mathrm{gen}} \mathbf{v}+\mathbf{i}_{o}
$$

Because there is no coupling between odd and even harmonics, vectors $\mathbf{i}$ and $\mathbf{v}$ and the generator admittance matrix $\mathbf{Y}_{\text {gen }}$ contain only the odd harmonics of $\mathbf{Y}_{\mathrm{abc}}$.

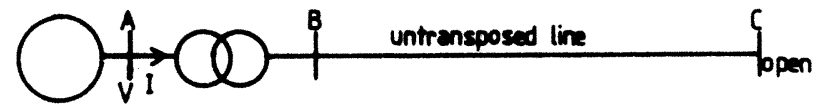

Figure 3 Test system

The input impedance matrix of the system $\left(Z_{s}\right)$, as seen from the generator bus, can be derived from existing programs [4], [7], so that for each harmonic the following relationship applies

$$
\mathbf{v}_{h}=\mathbf{Z}_{s_{h}} \mathbf{i}_{h}
$$

and for all harmonics

$$
\mathbf{v}=\mathbf{Z}_{s} \mathbf{i}
$$

From equation (32)

$$
\mathbf{i}_{o}=\mathbf{Y}_{\mathrm{gen}} \mathbf{v}+\mathbf{i}
$$

However, when the excitation is perfectly smooth, $i_{o}$ is only affected by positive sequence fundamental frequency current. Therefore equation (35) becomes

$$
\mathbf{i}_{o}=\mathbf{Y}_{\mathrm{gen}} \mathbf{v}_{+}+\mathbf{i}_{+}
$$

where $\mathbf{v}_{+}$and $\mathbf{i}_{+}$are vectors in the phase frame of reference containing only the positive sequence fundamental components of $\mathbf{v}$ and $\mathbf{i}$ respectively. While $\mathbf{v}_{+}$is kept fixed at 1 p.u., $i_{+}$is not known until the final solution. However $i_{+}$can be expressed as a function of $i$ using

$$
\mathbf{i}_{+}=\mathbf{T} \mathbf{i}
$$

where

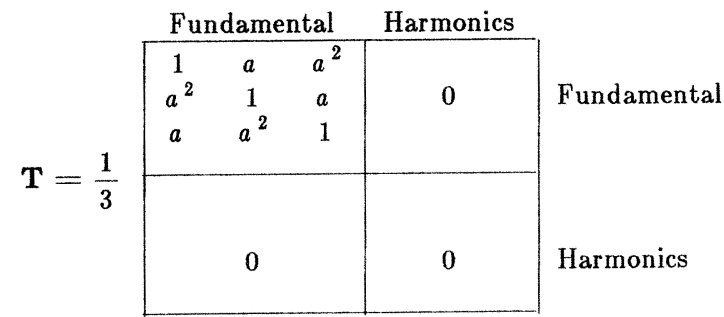

Combining equations (32), (34), (36) and (37) yields the following expression for the generator current

$$
\mathbf{i}=-\mathbf{Y}_{\mathrm{gen}} \mathbf{Z}_{8} \mathbf{i}+\mathbf{Y}_{\mathrm{gen}} \mathbf{v}_{+}+\mathbf{T} \mathbf{i}
$$

or

$$
\mathbf{A} \mathbf{i}=\mathbf{b}
$$

where

$$
\mathbf{A}=\mathbf{U}+\mathbf{Y}_{\text {gen }} \mathbf{Z}_{s}-\mathbf{T}
$$

and

$$
\mathbf{b}=\mathbf{Y}_{\mathrm{gen}} \mathbf{v}_{+}
$$

Equation (39) is solved for $\mathbf{i}$ by Gaussian elimination and $\mathbf{v}_{h}$ is obtained from (33) for each harmonic.

The transmission line introduces resonance and standing wave effects which somewhat obscure the understanding of the generator harmonic contribution. Thus the problem is analyzed in three stages, i.e. with the generator connected to (i) an unbalanced resistive dummy load, (ii) an unbalanced tuned load and (iii) an untransposed transmission line via a transformer. Details of the generator used in the test example are given in the Appendix.

\section{Unbalanced Resistive Load}

The load is represented by the following admittance matrix

$$
\mathbf{Y}_{o+-}=\left[\begin{array}{ccc}
Y_{o} & & \\
& Y_{+} & K Y_{+} \\
& K Y_{+} & Y_{+}
\end{array}\right]
$$

where $\mathbf{Z}_{8}$ is a block diagonal matrix 
where

$Y_{0}$ is a zero sequence conductance

$Y_{+}$is a positive (and negative) sequence conductance

$K$ is a coupling coefficient between the positive and negative sequences

Since the generator produces no zero sequence voltage, $Y_{0}$ can be chosen arbitrarily; to prevent a singularity its value is made equal to $Y_{+}$in our example. Thus the three-phase admittance matrix becomes

$$
Y_{a b c}=Y_{+}\left[\begin{array}{ccc}
1+\frac{2 K}{3} & -\frac{K}{3} & -\frac{K}{3} \\
-\frac{K}{3} & 1-\frac{K}{3} & \frac{2 K}{3} \\
-\frac{K}{3} & \frac{2 K}{3} & 1-\frac{K}{3}
\end{array}\right]
$$

This matrix, although not physically realizable, can be used to examine the effect of generator loading and degree of unbalance.

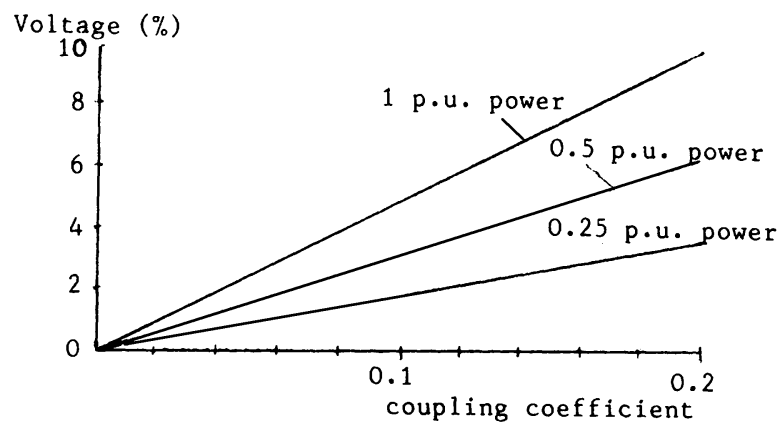

Figure 4 Variation of positive sequence third harmonic voltage with coupling and loading

Figure 4 shows the effect of coupling and loading on the level of 3rd harmonic voltage distortion. The results indicate that for loads within the nominal rating (i.e. 1 p.u.) the 3rd harmonic voltage is almost directly proportional to the load admittance, and hence to current or power. Also, the 3rd harmonic voltage is seen to be directly proportional to the level of coupling. The 5 th harmonic voltage, not shown in the figure, varies approximately in proportion to $K^{2}$.

\section{Effect of Saliency}

It is apparent from the analysis that saliency is the main determining factor in the process of harmonic conversion. To examine the effect of saliency, the simplified equivalent circuits in Figure 5 are used for the machine's direct and quadrature axes respectively. These circuits include only one winding in each axis of the rotor (i.e. $M_{d s}=M_{f_{s}}=0$ ). Further simplification is achieved by setting the direct axis magnetizing inductance $M_{d f}$ to 1 p.u. and making $L_{d}=L_{f}, L_{q}=L_{t}$ and $R_{f}=R_{t}$ and assuming equal leakage in both axes (i.e. $L_{d}-M_{d f}=L_{q}-M_{q t}$ ). Finally saliency, defined as the ratio of quadrature to direct axis rotor fluxes, can be expressed by

$$
S=1-\frac{M_{q t}}{M_{d f}}
$$

Thus from specified leakage, saliency and resistances $R$ and $R_{f}$, the machine parameters of equations (2) can be calculated.

While this model is not representative of an actual machine, it does provide a simple way of varying and displaying the effect of saliency on voltage distortion.

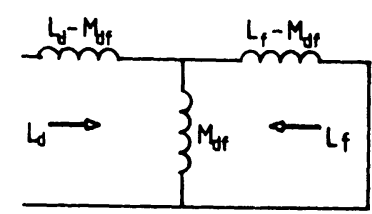

(a)

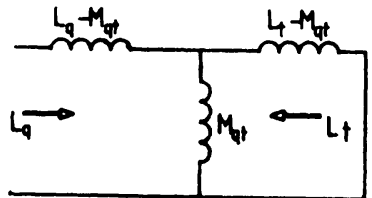

(b)
Figure 5 Simplified equivalent circuit of the machine (a) direct axis

(b) quadrature axis

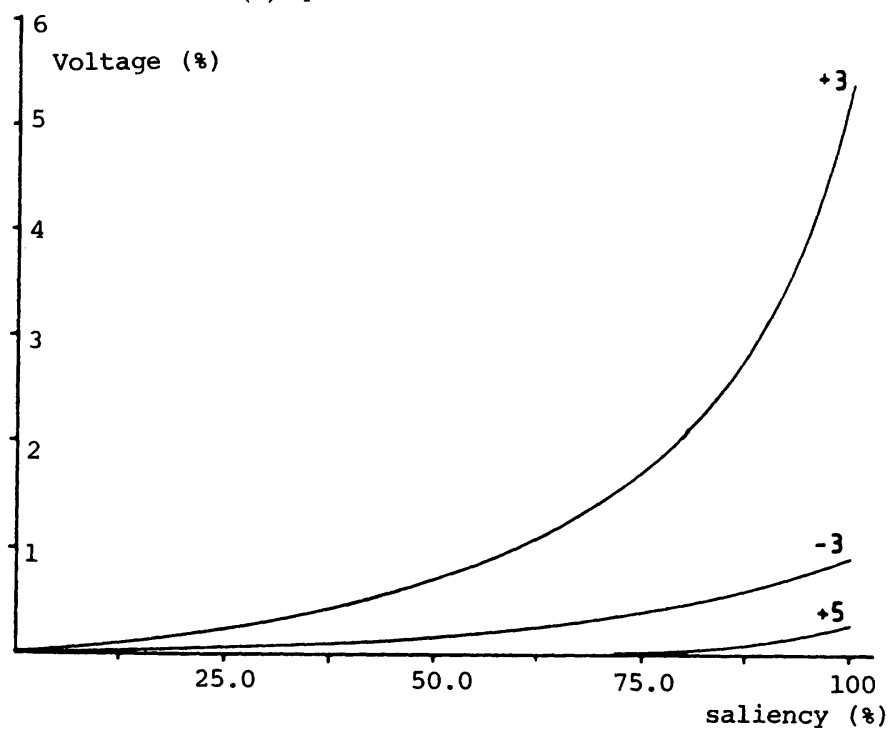

Figure 6 Effect of saliency on generator harmonic voltages

The effect of saliency has been tested on a purely resistive load of $484 \Omega(1$ p.u.) with a coupling coefficient of $10 \%$. Cases of perfect saliency $\left(M_{q t}=0\right)$ and zero saliency $\left(M_{q t}=1\right)$ produced no noticeable effect with perfect coupling (i.e. zero leakages). However, the addition of some leakage ( 0.2 p.u.) showed considerable difference in the resulting levels of harmonic voltage distortion, as shown in Figure 6.

\section{Unbalanced Tuned Load}

This case introduces the effect of resonance by replacing the resistive load with the delta connected circuit of Figure 7 , the resonance frequencies approximating those of the open circuited line of the test system. The machine data are given in the Appendix. cuit is

The sequence components admittance matrix of the delta cir-

$$
\mathbf{Y}_{o+-}=\left[\begin{array}{ccc}
0 & 0 & 0 \\
0 & (3+\delta) Y_{1} & -\delta a Y_{1} \\
0 & -\delta a^{2} Y_{1} & 3+\delta
\end{array}\right]
$$
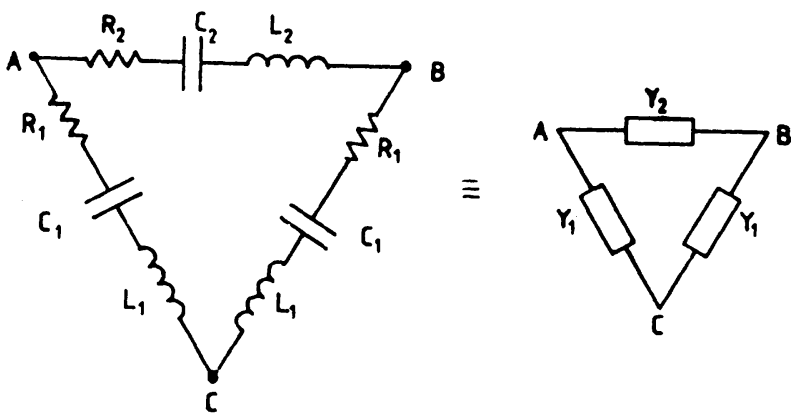

Figure 7 Tuned delta load 
where

$$
\begin{aligned}
& a=1\left\lfloor 120^{\circ}\right. \\
& Y_{2}=(1+\delta) Y_{1} \text {, valid at a particular frequency }
\end{aligned}
$$

and, using the previous definition of coupling coefficient,

$$
|K|=\left|\frac{\delta}{3+\delta}\right|
$$

The values of $L_{1}, C_{1}, L_{2}$ and $C_{2}$ are chosen to satisfy three conditions, namely, to give the same positive sequence admittance at the fundamental frequency, to produce specified resonance frequencies $f_{o 1}$ and $f_{o 2}$ for $Y_{1}$ and $Y_{2}$ respectively, and to maintain a specified degree of unbalance (determined by the coupling coefficient) at fundamental frequency. The three resistances are assumed equal.

From the test system with a line length of $200 \mathrm{~km}$, approximate values are derived for the delta branch parameters; at $50 \mathrm{~Hz}$ the reactance (mostly capacitive) and resistance are 1555 and 12 ohms, respectively.

In a real transmission line of flat construction the asymmetrical input impedance gives rise to two line modes which resonate at slightly different frequencies. This effect is simulated in the dummy load by maintaining a centre resonance frequency $f_{0}$ and varying the actual resonant frequencies of $Y_{1}$ and $Y_{2}$ symmetrically on both sides of $f_{0}$. Figure 8 shows the variation of harmonic voltages with $f_{01}-f_{02}$ while keeping $f_{0}$ constant at the third harmonic.

By maintaining a constant unbalance, the amount of negative sequence voltage and current at fundamental frequency is approximately constant with varying $f_{01}-f_{02}$.

With $f_{01}>f_{02} \quad Y_{1}$ is capacitive and $Y_{2}$ inductive and vice versa with $f_{02}>f_{01}$. This results in a strong unbalance at 3rd

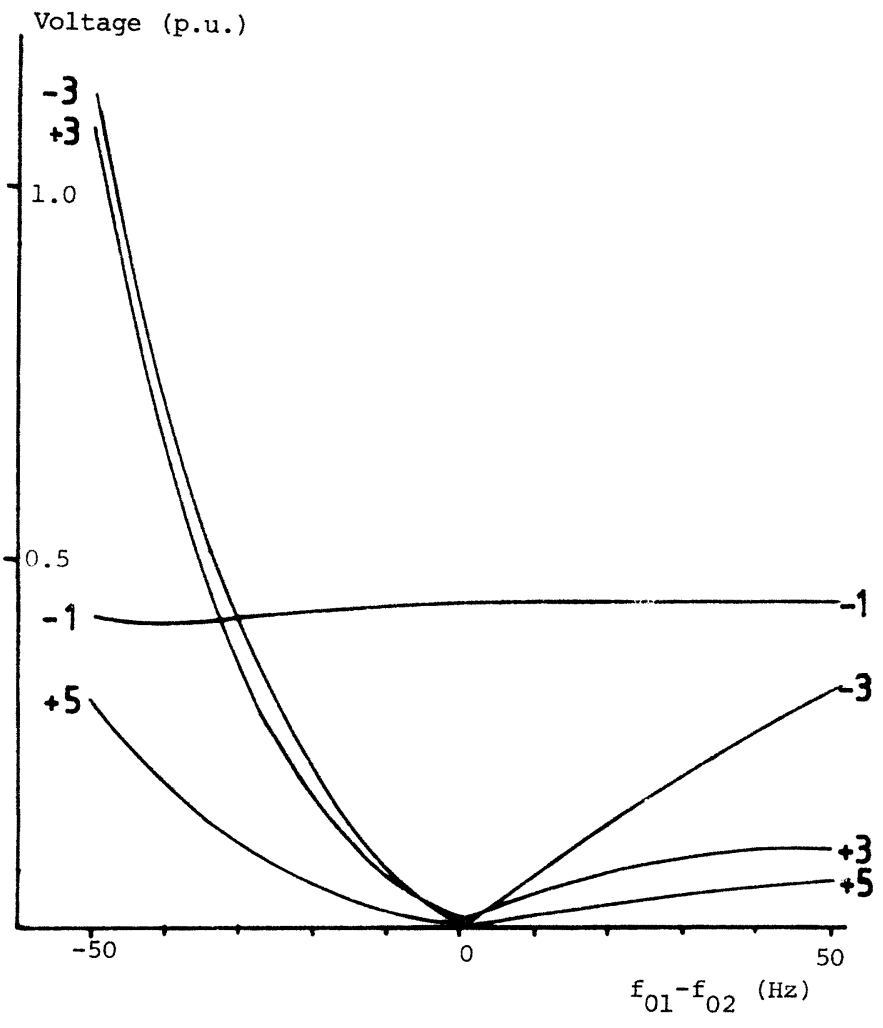

Figure 8 Harmonic voltage distortion versus $f_{01}-f_{02}$ with centre resonant frequency $f_{0}=150 \mathrm{~Hz}$ harmonic, leading to increasing negative sequence 3rd harmonic voltage and current with $f_{01}-f_{02}$. This is followed by corresponding increases of 5th harmonic voltage and current. The ratio of 5th harmonic positive sequence to $3 \mathrm{rd}$ harmonic negative sequence is approximately constant.

\section{Untransposed Open Circuit Line}

In the test system of Figure 3 the length of the untransposed line was varied from 50 to $800 \mathrm{~km}$, again with the machine dat of the Appendix.

Figures $9 \mathrm{a}$ and $\mathrm{b}$ show the positive and negative sequence third harmonic voltages at the machine terminals. While the machine cannot generate harmonics directly, these appear as a result of the unbalance produced by the untransposed line and are therefore very dependent on the length of line.

In the range of line lengths between 165 to $200 \mathrm{~km}$ each harmonic voltage shows a double peak. These peaks correspond to the different resonant lengths of the $\alpha$ and $\beta$ propagation modes present in a line of flat construction. The peaks occur at different lengths for the different harmonics (e.g. at 170 and $195 \mathrm{~km}$ for positive sequence third and at 175 and $190 \mathrm{~km}$ for negative sequence third). This effect is due to the impedances and degree of unbalance varying greatly near the resonances of the two modes.
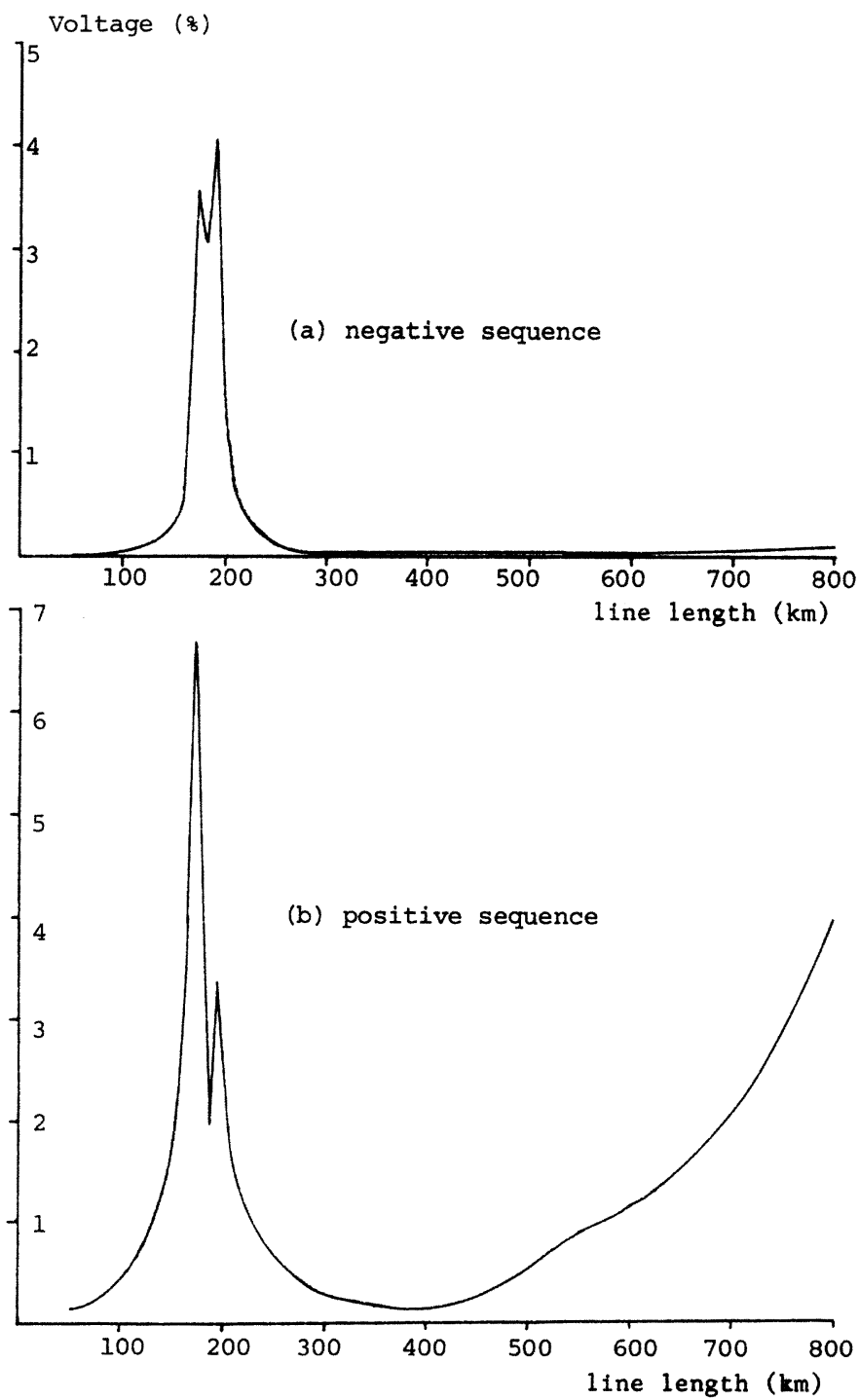

Figure 9 Variation of third harmonic voltage with transmission line length

(a) negative sequence

(b) positive sequence 
The harmonic voltage levels in this range of distances are too large to be ignored, viz. $\% \%$ of positive sequence third and $4 \%$ of negative sequence third harmonics respectively. This clearly demonstrates the need for detailed generator representation as proposed in this paper. The levels of distortion are also substantially higher than the levels calculated in the dummy loads, as a consequence of the standing wave effect of the line.

\section{CONCLUSIONS}

A generalized steady state model of the synchronous machine has been developed which can take into account any asymmetry or distortion present in the armature voltages. It has been shown that when the field voltage is perfect d.c. the harmonic model of the machine becomes a passive admittance matrix. The level of interharmonic coupling has proved to be partly load related (i.e. affected by the size of load current and degree of asymmetry) and partly generator related (affected by saliency). Such harmonic coupling cannot be detected with present harmonic models, where the generator is short-circuited behind the subtransient reactance.

Computer results, with the machine connected to a dummy (asymmetrical) load and to an untransposed transmission line, have been obtained to corroborate the theory. They indicate that the harmonics generated by the machine may often exceed the levels prescribed by harmonic legislation and they need to be assessed accurately. The effect of two different resonant modes have been demonstrated, leading to a strong unbalance and thus high voltage distortion. The main harmonic contributions from the generator are positive and negative sequence third harmonic currents, which therefore cannot be eliminated by generator or transformer connections.

\section{ACKNOWLEDGEMENTS}

The authors would like to express their appreciation to the Natural Sciences and Research Council of Canada and to the New Zealand Energy Research and Development Committee for financial support.

\section{REFERENCES}

[1] IEEE Working Group on Power System Harmonics, "Power System Harmonics: An Overview", IEEE Trans. on Power Apparatus and Systems, Vol. PAS-102, No. 8, Aug. 1983, pp. 2455-2460.

[2] W. Song, G.T. Heydt, and W.M. Grady, "The Integration of HVDC Subsystems into the Harmonic Power Flow Algorithm", ibidem, Vol. PAS-103, No. 8, Aug. 1984, pp. 19531961.
[3] D. Xia and G.T. Heydt, "Harmonic Power Flow Studies, Parts I and II", ibidem, Vol. PAS-101, No. 6, June 1982, pp. 1257-1270.

[4] T.J. Densem, P.S. Bodger, and J. Arrillaga, "Three Phase Transmission System Modeling for Harmonic Penetration Studies", ibidem, Vol. PAS-103, No. 2, Feb. 1984, pp. 310317.

[5] J. Arrillaga, D.A. Bradley, and P.S. Bodger, "Power System Harmonics", book, John Wiley, to appear in 1985.

[6] H.W. Dommel, A. Yan, and Shi Wei, "Harmonics from Transformer Saturation", IEEE Paper No. 85 SM 381-9, presented at the 1985 IEEE Summer Power Meeting, Vancouver, B.C.

[7] J. Arrillaga, T.J. Densem, and B.J. Harker, "Zero Sequence Harmonic Current Generation in Transmission Lines Connected to Large Converter Plant", IEEE Trans. on Power Apparatus and Systems, Vol. PAS-102, No. 7, July 1983, pp. 2357-2363.

[8] D. O'Kelly and S. Simmons, "Introduction to Generalized Machine Theory", book, McGraw-Hill, London, 1968.

[9] C.V. Jones, "The Unified Theory of Electrical Machines", book, Butterworths, London, 1967.

[10] H.H. Hwang, "Unbalanced Operation of Three-Phase Machines with Damper Circuits", IEEE Trans. on Power Apparatus and Systems, Vol. PAS-88, No. 11, November 1969, pp. 1585-1593.

\section{APPENDIX}

\section{Generator Data}

The generator data, based on that of Hwang [10], is as follows:

$$
\begin{aligned}
& \text { Nominal power rating }=100 \mathrm{MVA} \\
& \text { Nominal voltage }=14 \mathrm{kV} \\
& \text { Nominal frequency }=50 \mathrm{~Hz} \\
& R=0.005 \text { p.u. } \quad M_{d f}=1.0 \text { p.u. } \\
& R_{f}=0.0005 \text { p.u. } \quad M_{d s}=1.0 \text { p.u. } \\
& R_{\mathrm{o}}=R_{t}=0.02 \text { p.u. } \quad M_{q t}=0.6 \text { p.u. } \\
& L_{d}=1.2 \text { p.u. } \quad M_{f} \text {, }=1.0 \text { p.u. } \\
& L_{q}=0.8 \text { p.u. } \quad R_{o}=L_{o}=\infty \\
& L_{f}=1.2 \text { p.u. } \\
& L_{1}=1.0 \text { p.u. } \\
& L_{t}=0.831 \text { p.u. }
\end{aligned}
$$

